\title{
The NLRP3 Inflammasome as a promising target for Coronary Artery Disease: Current and Pipeline NLRP3 Inhibitors
}

\author{
Shauna Donnelly ${ }^{1}$, Roisin McAllister ${ }^{1}$, Melody Chemaly ${ }^{1}$, Tony Bjourson ${ }^{1}$, Aaron Peace ${ }^{2}$ and Victoria \\ McGilligan ${ }^{1 *}$ \\ ${ }^{1}$ Ulster University, Centre for Personalised Medicine, C-TRIC Clinical Translational Research and Innovation Centre, Altnagelvin Area Hospital, UK
}

${ }^{2}$ Department of Cardiology, Altnagelvin Area Hospital, UK

*Corresponding author: Victoria McGilligan, Ulster University, Centre for Personalised Medicine, C-TRIC Clinical Translational Research and Innovation Centre, Altnagelvin Area Hospital, UK.

Received Date: November 06, 2019

Published Date: November 13, 2019

\begin{abstract}
Coronary Artery Disease (CAD) represents a major health burden worldwide. It is driven by chronic inflammation of the arterial vasculature supplying the heart, in response to pro-inflammatory assaults such as high LDL cholesterol. The resultant atherosclerosis can cause occlusive disease and acute cardiovascular events. The pro-inflammatory cytokine, IL-1 $\beta$ is a central component of this inflammatory response and signalling is activated and amplified by the NLRP3 inflammasome. Rational therapeutic targeting of these mediators could modify atherosclerotic disease progression; myocardial remodelling and CAD patient outcomes. Here we discuss promising current and pipeline inhibitors of the NLRP3 inflammasome family.
\end{abstract}

Keywords: Coronary artery disease; Atherosclerosis; IL-1 $\beta$; NLRP3 inflammasome; IL-1 $\beta$ inhibitors; NLRP3 inhibitors

Abbreviations: CAD: Coronary Artery Disease; PCI: Percutaneous Coronary Intervention; CABG: Coronary Artery Bypass Graft; PAMPS: PathogenAssociated Molecular Pattern Molecules; DAMPS: Damage-Associated Molecular Patterns; NLRP3 (NOD [Nucleotide Oligomerization Domain]-, LRR [Leucine-Rich Repeat]-, And PYD [Pyrin Domain]-Containing Protein 3); ASC: Apoptosis-Associated Speck-Like Protein Containing A CARD; NLRC4: NLR Family CARD Domain Containing 4; AMI: Acute Myocardial Infarction.

\section{Introduction}

Coronary artery disease (CAD) is the leading cause of death worldwide [1,2]. Atherosclerosis of the coronary arteries is the underlying pathogenic mechanism and is driven by inflammation within the arterial intima in response to stressors including oxidised LDL cholesterol (ox-LDL) [3,4]. IL-1 $\beta$ is a major molecular determinant of atherosclerosis and is activated within the NLRP3 inflammasome. Atheromatous plaque build-up; acute plaque rupture and arterial thrombosis result in myocardial ischaemia and acute myocardial infarctions (AMI) respectively [5]. The mainstays of current treatment are medical or surgical. Medical interventions include statins to lower LDL; anti-coagulation; anti-hypertensives and cardiac contractility modifiers to reduce strain on ischaemic, post-infarction and pathologically remodelled hearts. Surgical interventions (PCI: Percutaneous Coronary Intervention and CABG:
Coronary Artery Bypass Graft) aim to revascularize ischaemic myocardial tissue [6]. However, there exists a clinical need for more effective targeted therapies to curtail disease progression and prevent recurrent events. Targeted anti-inflammatory agents are gaining traction in many diseases with an inflammatory basis, including in CAD, where IL-1 $\beta$ and/or NLRP3 inhibitors represent promising candidates [5,7].

\section{IL-1 $\beta$ role in CAD}

IL-1 $\beta$ is a key cytokine mediator of the response to infection and inflammation [8]. It plays an important role during all stages of atherosclerosis and CAD development, acting both within the vasculature wall and systemically [9]. Levels have been shown to correlate with atherosclerosis progression [10]. IL-1 $\beta$ modifies cholesterol metabolism [11], augmenting ox-LDL synthesis and 
promotes immune cell extravasation into the arterial intima during early plaque formation. Phagocytosis of ox-LDL by recruited macrophages forms engorged "foam cells" that secrete IL-1ß. Vascular smooth muscle cell proliferation is promoted, along with IL-6 expression from multiple cell types. Increased vascular inflammation contributes to plaque fibrosis; destabilisation and the rupture of vulnerable atherosclerotic plaques $[4,5]$. The acute phase response is induced (along with CRP) and the blood coagulation cascade is triggered, precipitating acute cardiovascular events [12]. IL-1 $\beta$ further plays a role in the pathological remodelling of the myocardium [13]. Subsequent to ischaemia-reperfusion, acute and chronic phases of inflammation and IL-1 $\beta$ expression drive the fibrosis and cell death known to permanently damage the myocardial tissue and cause heart failure [14].

\section{NLRP3 Inflammasome in CAD}

Inflammasomes are cytoplasmic oligomeric signalling complexes, formed within inflammatory cells, cardiomyocytes and endothelial cells in response to invading pathogens (PAMPs: Pathogen-Associated Molecular Patterns) and endogenous danger signals such as ox-LDL and cholesterol crystals (DAMPs: DamageAssociated Molecular Patterns). The NLRP3 (NOD [nucleotide oligomerization domain]-, LRR [leucine-rich repeat]-, and PYD [pyrin domain]-containing protein 3) inflammasome is the best characterised, and comprises NLRP3 (the sensing receptor); ASC (the adaptor) and caspase-1 (the effector that cleaves IL-1 $\beta$ and IL-18 pro-polypeptides) amplifying the inflammatory response
$[15,16]$. NLRP3 inflammasome activation is rate limited by the basally low levels of NLRP3 $m R N A$ maintained within cells [17]. Activation proceeds in a two-step manner. Firstly, a priming step induces NF-KB-mediated transcription of the NLRP3 and IL1B genes. Various lipid agonists, including LPS (lipopolysaccharide) and FFAs (free fatty acids) bind to TLRs and signal through the adaptor Myd88 and ox-LDL can induce signal one through a CD36/ TLR4/6 complex. TNF $\alpha /$ TNFR binding can further induce NF-KB activation. The second signal is required for NLRP3 component assembly and can be derived from intracellular molecules including ROS (reactive oxygen species) and cholesterol derivatives, degraded within the lysosome $[16,18]$. Assembly is further dependent on ATP, released from necrotic cells, binding the P2X7R and inducing $\mathrm{K}+$ ion efflux. Recruitment of ASC (apoptosis-associated speck-like protein containing a CARD) facilitates pro-caspase- 1 proximity induced activation to caspase- 1 and cleavage of pro- IL- $1 \beta$ and proIL-18 [19]. These cytokines then activate other immune cells to propagate the immune response. IL-1 $\beta$ binding to its cognate IL1R provides a feed-forward amplification loop. Pyroptopsis is a form of cell death activated by ischaemia and mitochondrial damage during AMI [20]. It releases intracellular molecules (such as ATP) further promoting NLRP3 activation. Consequently, a cycle of NLRP3 activation within the atheroma leads to chronic low-grade inflammation and advanced plaque formation and atherosclerotic plaques are associated with high levels of NLRP3 $m R N A$ expression [21] (Figure 1).

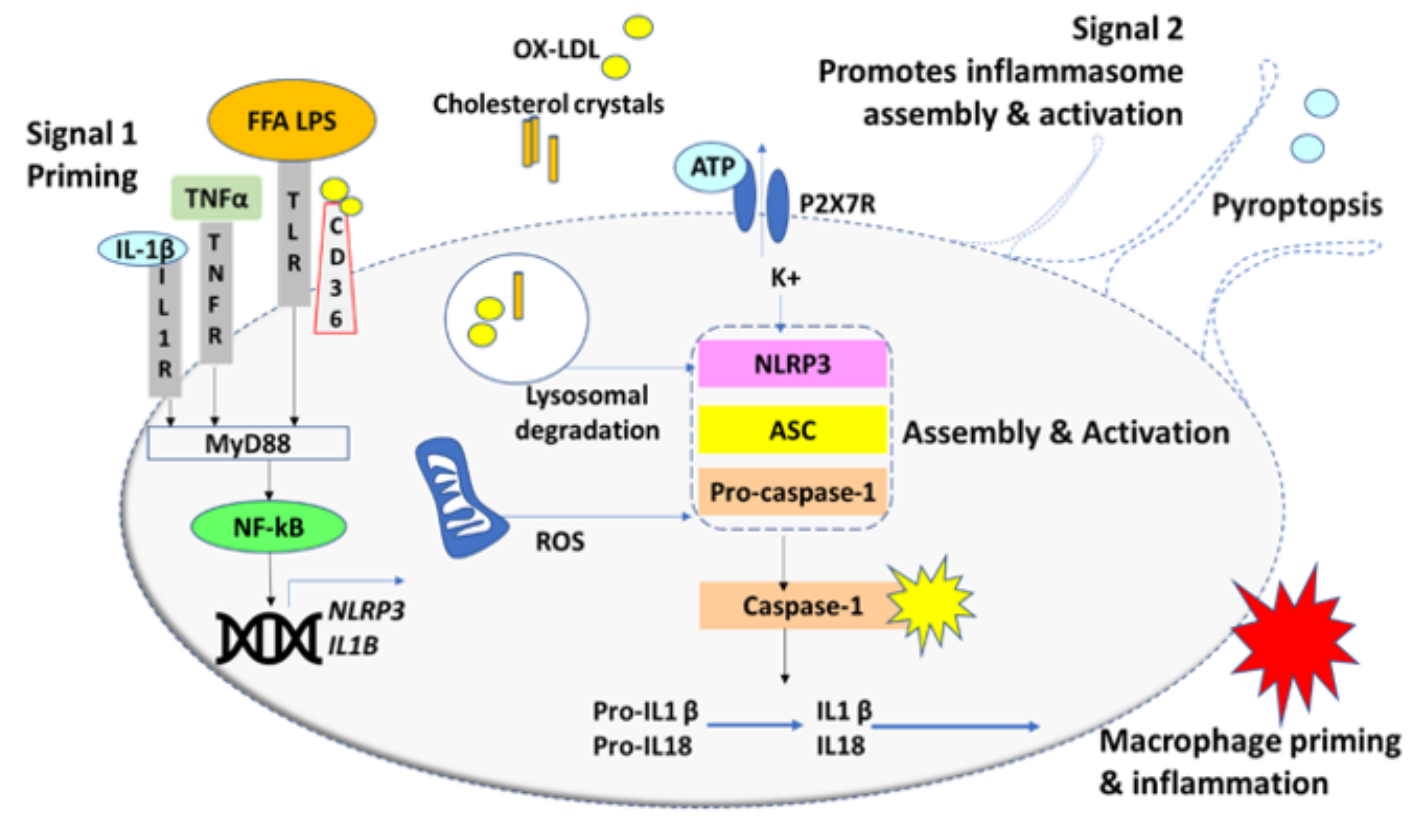

Figure 1: NLRP3 inflammasome two-step activation: The first priming step is initiated by lipid and lipoprotein endogenous danger signals and pathogen associated molecules (FFA: free fatty acids; ox-LDL: oxidised low density lipoprotein; cholesterol crystals; LPS: lipopolysaccharide) and cytokines (IL-1 $\beta$ and TNF $\alpha$ ) that signal through MyD88 to activate NF-KB mediated upregulation of NLRP3 and IL1B mRNA. The second signal is provided by diverse intracellular and extracellular molecules (ROS: reactive oxygen species released from mitochondria; lysosomally degraded ox-LDL and cholesterol crystals) and extracellular ATP binding to the $\mathrm{K}+$ channel P2X7R. This initiates NLRP3 inflammasome component (NLRP3; ASC; Pro-caspase-1) assembly and activation of caspase-1 to cleave the pro-peptides and release IL-1ß and IL-18. These mediators then prime other immune cells. Intracellular molecules are also released by pyroptopsis. 


\section{Inhibitors of the IL-1 $\beta$ Pathway}

Due to its central role in atherosclerosis, targeting the IL-1 $\beta$ pathway is a plausible approach for CAD therapeutic management. Many IL-1 $\beta$ inhibitors have been investigated in recent years in many diseases that have an underlying inflammatory component [22]. Anakinra is a recombinant human IL-1 competitive receptor antagonist FDA approved for rheumatoid arthritis treatment. Its utility is, however, reduced by its biological half-life (4hrs) $[23,24]$. Canakinumab is a recombinant human monoclonal antibody that selectively inhibits IL-1 $\beta$ receptor binding [25]. The CANTOS clinical trial supported the role of atherosclerosis in CAD and served as a proof of concept that an IL-1 $\beta$ inhibitor could influence the trajectory of atherosclerosis development without plasma cholesterol reduction [26,27]. The use of canakinumab is

Table 1: Summary of IL-1 $\beta$ inhibitors.

\begin{tabular}{|c|c|c|c|c|}
\hline IL-1 $\beta$ inhibitor & Mode of inhibition & Tested in context of CAD & CAD Clinical trial & References \\
\hline Anakinra & $\begin{array}{l}\text { Recombinant human IL-1 competitive receptor } \\
\text { antagonist }\end{array}$ & Yes & $\begin{array}{l}\text { Yes (Phase II - no results } \\
\text { posted) }\end{array}$ & {$[24,69]$} \\
\hline Canakinumab & Selectively inhibits IL- $1 \beta$ receptor binding & Yes & $\begin{array}{l}\text { Yes (Phase III - no results } \\
\text { posted) }\end{array}$ & {$[26,70]$} \\
\hline Rilonacept & $\begin{array}{l}\text { Decoy receptor antagonist of IL- } 1 \text { that binds } \\
\text { with a high affinity to IL- } 1 \beta \text { and blocks its } \\
\text { activity }\end{array}$ & Yes & $\begin{array}{l}\text { Yes (Phase II - results } \\
\text { posted) }\end{array}$ & {$[31,71]$} \\
\hline Gevokizumab & $\begin{array}{l}\text { Decreases the binding affinity of IL- } 1 \beta \text { for the } \\
\text { IL-1 receptor type I (IL-1RI) signalling receptor }\end{array}$ & Yes & No & [72] \\
\hline
\end{tabular}

\section{Inhibitors of the NLRP3 Inflammasome}

The NLRP3 inflammasome amplifies the local inflammatory response within the arterial intima and myocardium, in a feedforward signalling loop to produce IL-1 $\beta$. Therefore, inhibition of this inflammasome might quell IL-1 $\beta$ inflammatory signalling; without complete IL-1 $\beta$ pathway blockade and accompanying susceptibility to infection. Further, NLRP3 targeting is potentially more specific than inhibiting IL-1 $\beta$, where inhibitor off-target binding to IL- $1 \alpha$ can further dampen the immune response to pathogens.

\section{Non-specific Inflammasome Inhibitors}

Several known drugs have non-specific NLRP3 inflammasome inhibition properties. Arglabin is a sesquiterpene lactone antitumor agent isolated from Artemisia species [34]. Pre-clinical studies demonstrated NLRP3 inhibition in macrophages that were exposed to cholesterol crystals and attenuation of atherosclerotic progression in ApoE2.ki mice on a high fat diet [35]. Difficulties in large-scale production of this agent would hinder its clinical use [36]. Atorvastatin, the most commonly used cholesterol reduction therapy, is a 3-hydroxyl-3-methylglutaryl coenzyme A inhibitor that has anti-inflammatory properties [37]. It inhibits NLRP3 activation via TLR4/MyD88/NF-KB signalling in PMA-stimulated THP-1 monocytes and halts atherosclerotic plaque progression in patients receiving high-dose atorvastatin [38]. Other statin analogues display similar properties [39]. Studies have shown mild adverse events associated with atorvastatin such as mild gastrointestinal complaints and central nervous effects, mainly headaches [40]. Colchicine derived from Colchicum autumnale is used for the restricted nevertheless as it can cause fatal opportunistic infections and sepsis [28]. Gevokizumab showed better tolerance in Phase II clinical trials [29]. Rilonacept is a soluble decoy receptor antagonist of IL- 1 that binds with a high affinity to IL- $1 \beta$ and blocks its activity; but has also displayed adverse reactions including injection site reactions and infections [30]. Therefore, targeting inflammatory signalling at the level of IL-1 $\beta$ may not be the optimal level for pathway attenuation in atherosclerosis $[31,32]$. IL-1 $\beta$ is processed by multiple types of inflammasomes (including NLRC4), especially in response to pathogenic assaults [33]. Targeting upstream of IL- $\beta$ by specifically targeting NLRP3 the main orchestrator of IL$1 \beta$ activation presents an attractive option, thereby leaving other methods of IL-1 $\beta$ production to respond to severe infection if required (Table 1 ).

treatment of gout [41]. Recent studies have shown it is capable of inhibiting NLRP3 inflammasome activation by interfering with its oligomerisation [42]. In clinical trials it reduced CRP levels in patients with stable CAD and it may possess a protective effect in stable CAD [43]. However, it is prone to drug-drug interactions and off-target effects, reducing its clinical potential [44]. Glyburide is a sulfonylurea-based compound used to treat type 2 diabetes. In diabetes it inhibits ATP-dependent $\mathrm{K}+$ channels to augment insulin secretion [45]. Its inhibition of the NLRP3 inflammasome however is independent of $\mathrm{K}+$ channel activation and its molecular target is unknown. A further drawback is that this inhibitor has a relatively high $\mathrm{IC}_{50}$ value $(100 \mu \mathrm{M}$ in trophoblasts) increasing the chance of off-target effects [46]. Natural metabolites produced by the body can also inhibit the NLRP3 inflammasome. The ketone body $\beta$-hydroxybutyrate (BHB) is an alternative source of ATP in mammals during states of energy deficit. BHB can reduce NLRP3 inflammasome-mediated interleukin IL-1 $\beta$ and IL-18 production in human monocytes. In mice BHB or a ketogenic diet attenuates caspase-1 activation and IL-1 $\beta$ secretion, demonstrating its potential to curtail NLRP3-mediated diseases [47].

\section{Specific and Pipeline Inflammasome Inhibitors}

Specific, small molecule NLRP3 inhibitors have generally been identified through phenotypic screening for bioactivities. However, due to the complexity of the NLRP3 activation pathway; inhibition may be direct or via accessory proteins and the mechanistic basis of their activity is mostly elusive. CP-456,773 (MCC950) is the best characterised NLRP3 inhibitor. It is another sulfonylureacontaining compound and small molecule that is a highly specific 
NLRP3 inflammasome inhibitor, that inhibits NLRP3 ATPase activity and attenuates IL-1 $\beta$ secretion by all known activators (including ATP) [48]. Apolipoprotein E-deficient mice treated with MCC950, showed a reduction in atherosclerotic plaques by reduction in plaque monocyte adhesion molecules [49]. Clinical use will, however, be limited by the molecule's short half-life (3.27hrs) and the associated drawbacks of daily injections and site reactions [50]. OLT1177, a $\beta$-sulfonyl nitrile compound has been shown to be safe in humans and is another selective NLRP3 inhibitor [51]. Very recently it was reported that OLT1177 (dapansutrile) limits infarct size and prevents left ventricular systolic dysfunction when given within 60 minutes following ischemia reperfusion injury in the mouse [52]. 16673-34-0 is a small molecule inhibitor and an intermediate substrate in glyburide synthesis. It inhibits the formation of the NLRP3 inflammasome in cardiomyocytes and limits the infarct size after myocardial ischemia-reperfusion in a mouse model [53]. Tranilast is an old anti-allergic drug, recently been shown to be a specific NLRP3 inflammasome inhibitor by preventing its oligomerisation [54] and was have shown Tranilast to reduce atherosclerotic plaque development [55]. Further novel agents are being developed; although not yet tested in CAD. CY09 was identified through structure-activity relationship studies and was shown to inhibit NLRP3 ATPase activity. In mice fed a high fat diet, CY-09 reduced blood glucose and insulin levels [56] Other potent NLRP3 inhibitors such as NT-0167 (NodThera ${ }^{\mathrm{TM}}$ ) are being trialled in fibrosis [57]. Inflammasome Therapeutics Inc $^{\mathrm{TM}}$. have identified kamuvudines that are chemical derivatives of Nucleoside Reverse Transcriptase Inhibitors (NRTIs), the mainstay of HIV treatment regimens. These agents inhibit P2X7Rmediated NLRP3 inflammasome activation independent of targeting reverse transcriptase. They are currently being tested in macular degeneration and type 2 diabetes [58,59]. Other agents can target the NLRP3 inflammasome at the level of ASC. IC100 is a monoclonal antibody developed by Zyversa Therapeutics ${ }^{\mathrm{TM}}$ that inhibits the adaptor ASC component of multiple inflammasomes, offering the potential to treat a broad group of inflammatory disorders, notably multiple sclerosis [60]. However, since ASC is common to many inflammasome species, the risk of non-specificity and infections remains problematic. Nevertheless, the avenue of ASC inhibition warrants exploration in CAD. The development of biologic agents (such as IC100) offers the benefits of enhanced specificity and longer therapeutic half-lives over the small molecule inhibitors. They can, however, be more expensive to produce and cell penetrability can be an issue [61,62]. There are reports of novel ways being developed to assist entry of antibodies inside the cell [63] (Table 2).

Table 2: Summary of current and pipeline NLRP3 inhibitors.

\begin{tabular}{|c|c|c|c|c|}
\hline NLRP3 inhibitor & Mode of Inhibition & $\begin{array}{l}\text { Tested in } \\
\text { context of CAD }\end{array}$ & CAD Clinical trial & References \\
\hline Atrovastatin & $\begin{array}{c}\text { Selectively inhibits NLRP3 activation-- } \\
\text { suppresses TLR4/MyD88/NF-кB pathway }\end{array}$ & Yes & $\begin{array}{l}\text { Yes (phase IV - no results } \\
\text { posted) }\end{array}$ & {$[37,73]$} \\
\hline Colchicine & $\begin{array}{l}\text { Inhibits NLRP3 by disrupting } \beta \text {-tubulin } \\
\text { polymerization into microtubules and inhibits } \\
\text { adhesion molecules }\end{array}$ & No & $\begin{array}{l}\text { Yes (Phase II - no results } \\
\text { posted) }\end{array}$ & {$[43,74]$} \\
\hline Arglabin & $\begin{array}{c}\text { Selectively inhibits NLRP3-induces } \\
\text { autophagy and reduces cholesterol levels and } \\
\text { inflammation }\end{array}$ & Yes & No & [36] \\
\hline MCC950 & $\begin{array}{c}\text { Interacts with NLRP3 and blocks ATP } \\
\text { hydrolysis-inhibits NLRP3 activation and } \\
\text { formation }\end{array}$ & Yes & No & [49] \\
\hline OLT1177 & $\begin{array}{l}\text { Selective NLRP3 inhibitor, } \beta \text {-Sulfonyl nitrile } \\
\text { compound. }\end{array}$ & Yes & Yes (Phase Ib) & {$[51,52]$} \\
\hline Tranilast & $\begin{array}{l}\text { Directly binds to NLRP3 and inhibits } \\
\text { oligomerization }\end{array}$ & Yes & No & [55] \\
\hline BHB & $\begin{array}{c}\text { Supresses NLRP3 in response to urate crystals, } \\
\text { ATP and lipotoxic fatty acids - prevents K+ } \\
\text { efflux }\end{array}$ & No & No & [48] \\
\hline CY-09 & $\begin{array}{l}\text { Inhibition of NLRP3 ATPase and } \\
\text { oligomerisation }\end{array}$ & No & No & {$[57]$} \\
\hline Glyburide & $\begin{array}{l}\text { Independent of } \mathrm{K}+\text { channel activation. } \\
\text { Molecular target unknown }\end{array}$ & No & No & {$[46]$} \\
\hline IC 100 & Inhibits ASC component & No & No & [61] \\
\hline NT-0167 & A potent selective NLRP3 inhibitor & No & No & [58] \\
\hline 16673-34-0 & Inhibits NLRP3 formation in cardiomyocytes & No & No & {$[54]$} \\
\hline Kamuvudines & $\begin{array}{l}\text { Inhibits P2X7R-mediated NLRP3 inflammasome } \\
\text { activation }\end{array}$ & No & No & {$[57,58]$} \\
\hline
\end{tabular}




\section{Conclusion}

There exists a salient need for therapeutics capable of modifying CAD progression and for improving clinical outcomes. Cholesterol lowering statins are the mainstay of treatment; but plaque progression occurs in most patients irrespective of lowered cholesterol levels [63]. The chronic nature of the disease lends itself to anti-inflammatory pharmacological management. Targeting the inflammation driving atherosclerosis is a rational approach with demonstrated success in the seminal CANTOS trial of IL-1 $\beta$ inhibitor canakinumab. In a large patient cohort with stable CAD, inflammatory markers (including IL-6 and CRP) were lowered and the risk of an acute event or a recurrent event was reduced, independent of cholesterol lowering. This provides proof of concept that dampening of atherosclerotic chronic inflammation could augment existing statin lowering therapies [39]. However, targeting IL-1 $\beta$ directly may be not be the optimal strategy; as it could interfere with systemic immune homeostasis. Susceptibility to infection is a limitation that may reduce the clinical utility of such agents [64]. The NLRP3 inflammasome activates and amplifies IL$1 \beta$ signalling by integrating multiple pathogen and endogenous danger signals. It is an established mediator of atherosclerotic plaque formation and progression and acts locally to activate and amplify the inflammatory response. It may therefore be a more specific target. Known drugs, including glyburide (an antidiabetic agent), have NLRP3 inhibitory properties; although predicable off-target effects and lack of efficacy may limit their use in CAD. Specific inhibitors are largely being identified through phenotypic screens and often the mechanism of action is unclear or unknown; although many specifically target NLRP3 activation at the level of oligomerisation. Testing so far has been pre-clinical. Encouraging results in mouse studies have shown that reduction in markers of NLRP3 activation (caspase-1, IL-1 $\beta$ ) correlate with a reduction in plaque progression. Abderrazak et al. [35] found that arglabin could reduce plaque progression in mice genetically engineered to have high blood cholesterol. MCC950 is a potent and specific NLRP3 inhibitor with similar properties [49]. If initiated early enough; such agents could maintain atherosclerosis at a sub-clinical level. These agents may also have clinical utility in reducing the damage heart muscle sustains post-myocardial infarction. In mice, 16673-34-0 was shown to reduce infarct size after myocardial ischemia-reperfusion [53]. However, caution is always required when extrapolating mouse studies into humans [65]. The detailed mechanisms of NLRP3 activation in response to activating signals and its negative regulation are still being fully elucidated [66]. Additionally, no X-ray crystallographic structures have been resolved for the NLRP3 components, limiting rational drug design [67]. Filling in these knowledge gaps will facilitate the development of more specific agents. Currently, the targeting of other accessory proteins such as ASC is being explored. Still, many of these agents are encumbered with the problem of short half-lives and site injection reactions. Improved drug formulations and pharmacokinetics will help circumvent the issues. BHB has been shown to reduce NLRP3 activation [47], demonstrating the potential for non-pharmacologic interventions. Excessive alcohol consumption is a known cardiovascular disease risk factor [68]; that may also inhibit NLRP3 activation [69] Therefore, it is also encouraging that lifestyle changes, in the form of ketogenic diets and modified ethanol consumption, may provide a drug-free alternative to CAD outcomes [70-75].

\section{Acknowledgements}

This work was supported by Invest Northern Ireland.

\section{Conflict of Interest}

No conflict of interest

\section{References}

1. Lozano R, Naghavi M, Foreman K, Lim S, Shibuya K, et al. (2012). Global and regional mortality from 235 causes of death for 20 age groups in 1990 and 2010: a systematic analysis for the Global Burden of Disease Study 2010. Lancet 380: 2095-2128.

2. Khera A, Kathiresan S (2017) Genetics of coronary artery disease: discovery, biology and clinical translation. Nature Reviews Genetics 18(6): 331-344

3. van der Wal AC, Becker AE (1999) Atherosclerotic plaque rupture - pathologic basis of plaque stability and instability. Cardiovascular Research 41(2): 334-344.

4. (2019) Atherosclerosis. Nature Reviews Disease Primers 5(1): 56.

5. Grebe A, Hoss F, Latz E (2018) NLRP3 Inflammasome and the IL-1 Pathway in Atherosclerosis. Circulation Research 122(12): 1722-1740.

6. nhs uk (2019) Coronary heart disease - Treatment.

7. Shao B, Xu Z, Han B, Su D, Liu C, et al. (2015) NLRP3 inflammasome and its inhibitors: a review. Frontiers in Pharmacology 6: 262.

8. Dinarello C (2017) Overview of the IL-1 family in innate inflammation and acquired immunity. Immunological Reviews 281(1): 8-27.

9. Szekely Y, Arbel Y (2018) A Review of Interleukin-1 in Heart Disease: Where Do We Stand Today. Cardiology and Therapy 7(1): 25-44.

10. Van Tassell BW, Toldo S, Mezzaroma E, Abbate A (2013) Targeting Interleukin-1 in Heart Disease. Circulation 128(17): 1910-1923.

11. Persson J, Nilsson J, Lindholm MW (2008) Interleukin-1beta and tumour necrosis factor-alpha impede neutral lipid turnover in macrophagederived foam cells. BMC Immunology 9(1): 70.

12. Maier W, Altwegg LA, Corti R, Gay S, Hersberger M, et al. (2005) Inflammatory Markers at the Site of Ruptured Plaque in Acute Myocardial Infarction. Circulation 111(11): 1355-1361.

13. Van Tassell BW, Toldo S, Mezzaroma E, Abbate A (2013) Targeting Interleukin-1 in Heart Disease. Circulation 128(17): 1910-1923.

14. Harouki N, Nicol L, Remy Jouet I, Henry J, Dumesnil A, et al. (2017) The IL-1 $\beta$ Antibody Gevokizumab Limits Cardiac Remodeling and Coronary Dysfunction in Rats with Heart Failure. JACC: Basic to Translational Science 2(4): 418-430.

15. He Y, Hara H, Núñez G (2016) Mechanism and Regulation of NLRP3 Inflammasome Activation. Trends in Biochemical Sciences 41(12): 1012-1021.

16. Malik A, Kanneganti TD (2017) Inflammasome activation and assembly at a glance. Journal of Cell Science 130(23): 3955-3963.

17. Abais JM, Xia M, Zhang Y, Boini KM, Li PL, et al. (2015) Redox Regulation of NLRP3 Inflammasomes: ROS as Trigger or Effector. Antioxidants \& Redox Signaling 22(13): 1111-1129.

18. Yang Y, Wang H, Kouadir M, Song H, Shi F, et al. (2019) Recent advances in the mechanisms of NLRP3 inflammasome activation and its inhibitors. Cell Death \& Disease 10(2): 128. 
19. Compan V, Martín-Sánchez F, Baroja-Mazo A, López-Castejón G, Gomez AI, et al. (2014) Apoptosis-Associated Speck-like Protein Containing a CARD Forms Specks but Does Not Activate Caspase-1 in the Absence of NLRP3 during Macrophage Swelling. The Journal of Immunology 194(3): 1261-1273.

20. Zha Q Wei H, Li C, Liang Y, Xu L, et al. (2016) ATP-Induced Inflammasome Activation and Pyroptosis Is Regulated by AMP-Activated Protein Kinase in Macrophages. Frontiers in Immunology 7: 597.

21. Zeng C, Wang R, Tan H (2019) Role of Pyroptosis in Cardiovascular Diseases and its Therapeutic Implications. International Journal of Biological Sciences 15(7): 1345-1357.

22. Dinarello CA, Simon A, van der Meer JW (2012) Treating inflammation by blocking interleukin-1 in a broad spectrum of diseases. Nature Reviews Drug Discovery 11(8): 633-652.

23. Baskar S, Klein AL, Zeft A (2016) The Use of IL-1 Receptor Antagonist (Anakinra) in Idiopathic Recurrent Pericarditis: A Narrative Review. Cardiology Research and Practice pp.1-6.

24. Kaiser C, Knight A, Nordström D, Pettersson T, Fransson J, et al. (2011) Injection-site reactions upon Kineret (anakinra) administration: experiences and explanations. Rheumatology International 32(2): 295299.

25. Dhimolea E (2010) Canakinumab. mAbs 2(1): 3-13.

26. Lorenzatti A, Servato M (2018) Role of Anti-inflammatory Interventions in Coronary Artery Disease: Understanding the Canakinumab Antiinflammatory Thrombosis Outcomes Study (CANTOS). European Cardiology Review 13(1): 38-41.

27. Ridker PM, Everett BM, Thuren T, MacFadyen JG, Chang WH, et al. (2017) Antiinflammatory Therapy with Canakinumab for Atherosclerotic Disease. New England Journal of Medicine 377(12): 1119-1131.

28. Ridker PM, Thuren T, Zalewski A, Libby P (2011) Interleukin-1ß inhibition and the prevention of recurrent cardiovascular events: Rationale and Design of the Canakinumab Anti-inflammatory Thrombosis Outcomes Study (CANTOS). American Heart Journal 162(4): 597-605.

29. Geiler J, McDermott MF (2010) Gevokizumab, an anti-IL-1 $\beta$ mAb for the potential treatment of type 1 and 2 diabetes, rheumatoid arthritis and cardiovascular disease. Current Opinion on Molecular Therapeutics 12(6): 755-769.

30. Dubois E, Rissmann R, Cohen A (2011) Rilonacept and canakinumab. British Journal of Clinical Pharmacology 71(5): 639-641.

31. Regeneron.com (2019).

32. White PC, Adhikari S, Grishman EK, Sumpter KM (2018) A phase I study of anti-inflammatory therapy with rilonacept in adolescents and adults with type 1 diabetes mellitus. Pediatric Diabetes 19(4): 788-793.

33. Próchnicki T, Latz E (2017) Inflammasomes on the Crossroads of Innate Immune Recognition and Metabolic Control. Cell Metabolism 26(1): 71 93.

34. Lone S, Bhat K, Khuroo M (2015) Arglabin: From isolation to antitumor evaluation. Chemico-Biological Interactions 240: 180-198.

35. Abderrazak A, Couchie D, Mahmood DF, Elhage R, Vindis C, et al (2015) Anti-Inflammatory and Antiatherogenic Effects of the NLRP3 Inflammasome Inhibitor Arglabin in ApoE2.Ki Mice Fed a High-Fat Diet. Circulation 131(12): 1061-1070.

36. Sülsen V, Martino V (2008) Sesquiterpene Lactones. p.129.

37. Eshtehardi P, McDaniel MC, Dhawan SS, Binongo JN, Krishnan SK, et al. (2012) Effect of Intensive Atorvastsain Therapy on Coronary Atherosclerosis Progression, Composition, Arterial Remodelling, and Microvascular Function. Journal of Invasive Cardiology, 24(10): 522529.

38. Kong F, Ye B, Lin L, Cai X, Huang W, et al. (2016) Atorvastatin suppresses NLRP3 inflammasome activation via TLR4/MyD88/NF- $\mathrm{kB}$ signaling in PMA-stimulated THP-1 monocytes. Biomedicine \& Pharmacotherapy 82: $167-172$
39. Wang S, Xie X, Lei T, Zhang K, Lai B, et al. (2017) Statins Attenuate Activation of the NLRP3 Inflammasome by Oxidized LDL or TNFa in Vascular Endothelial Cells through a PXR-Dependent Mechanism. Molecular Pharmacology 92(3): 256-264.

40. Posvar EL, Radulovic LL, Cilla DD Jr, Whitfield LR, Sedman AJ, et al. (1996) Tolerance and Pharmacokinetics of Single-Dose Atorvastatin, a Potent Inhibitor of HMG-CoA Reductase, in Healthy Subjects. The Journal of Clinical Pharmacology 36(8): 728-731.

41. Slobodnick A, Shah B, Krasnokutsky S, Pillinger MH (2017) Update on colchicine. Rheumatology 57(suppl_1): i4-i11.

42. Demidowich AP, Davis AI, Dedhia N, Yanovski JA (2016) Colchicine to decrease NLRP3-activated inflammation and improve obesity-related metabolic dysregulation. Medical Hypotheses 92: 67-73.

43. Nidorf M, Thompson P (2007) Effect of Colchicine (0.5 mg Twice Daily) on High-Sensitivity C-Reactive Protein Independent of Aspirin and Atorvastatin in Patients with Stable Coronary Artery Disease. The American Journal of Cardiology 99(6): 805-807.

44. Balasubramanian E, Gajendran T (2013) Comparative Studies on the Anticancer Activity of Colchicine by Various Controlled Drug Delivery Modes. International Journal of Pharma and Bio Sciences 4(1): 9-26.

45. Rendell M (2004) The Role of Sulphonylureas in the Management of Type 2 Diabetes Mellitus. Drugs 64(12): 1339-1358.

46. Tamura K, Ishikawa G, Yoshie M, Ohneda W, Nakai A, et al (2017) Glibenclamide inhibits NLRP3 inflammasome-mediated IL-1 $\beta$ secretion in human trophoblasts. Journal of Pharmacological Sciences 135(2): 8995.

47. Youm YH, Nguyen KY, Grant RW, Goldberg EL, Bodogai M, et al. (2015) The ketone metabolite $\beta$-hydroxybutyrate blocks NLRP3 inflammasomemediated inflammatory disease. Nature Medicine 21(3): 263-269.

48. Coll RC, Hill JR, Day CJ, Zamoshnikova A, Boucher D, et al. (2019) MCC950 directly targets the NLRP3 ATP-hydrolysis motif for inflammasome inhibition. Nature Chemical Biology 15(6): 556-559.

49. van der Heijden T, Kritikou E, Venema W, van Duijn J, van Santbrink PJ, et al. (2017) NLRP3 Inflammasome Inhibition by MCC950 Reduces Atherosclerotic Lesion Development in Apolipoprotein E-Deficient Mice-Brief Report. Arteriosclerosis Thrombosis and Vascular Biology 37(8): 1457-1461.

50. Coll RC, Robertson AA, Chae JJ, Higgins SC, Muñoz-Planillo R, et al. (2015) A small-molecule inhibitor of the NLRP3 inflammasome for the treatment of inflammatory diseases. Nature Medicine 21(3): 248-255.

51. Marchetti C, Swartzwelter B, Gamboni F, Neff CP, Richter K, et al. (2018) OLT1177, a $\beta$-sulfonyl nitrile compound, safe in humans, inhibits the NLRP3 inflammasome and reverses the metabolic cost of inflammation. Proc Natl Acad Sci U S A 115(7): E1530-E1539.

52. Toldo S, Mauro AG, Cutter Z, Van Tassell BW, Mezzaroma E, et al. (2019) The NLRP3 Inflammasome Inhibitor, OLT1177 (Dapansutrile), Reduces Infarct Size and Preserves Contractile Function After Ischemia Reperfusion Injury in the Mouse. J Cardiovasc Pharmacol 73(4): 215222.

53. Marchetti C, Chojnacki J, Toldo S, Mezzaroma E, Tranchida N et al. (2014) A Novel Pharmacologic Inhibitor of the NLRP3 Inflammasome Limits Myocardial Injury After Ischemia-Reperfusion in the Mouse. Journal of Cardiovascular Pharmacology 63(4): 316-322.

54. Huang Y, Jiang H, Chen Y, Wang X, Yang Y, et al. (2018) Tranilast directly targets NLRP 3 to treat inflammasome-driven diseases. EMBO Molecular Medicine 10(4).

55. Matsumura T, Kugiyama K, Sugiyama S, Ota Y, Doi H, et al. (1999) Suppression of Atherosclerotic Development in Watanabe Heritable Hyperlipidemic Rabbits Treated with an Oral Antiallergic Drug, Tranilast. Circulation 99(7): 919-924.

56. Jiang H, He H, Chen Y, Huang W, Cheng J, et al. (2017) Identification of a selective and direct NLRP3 inhibitor to treat inflammatory disorders. The Journal of Experimental Medicine 214(11): 3219-3238. 
57. NodThera (2019) NodThera Highlights Significant Progress with Advancement of Lead Development Candidate and Key Additions to Board - NodThera.

58. Inflam.com. (2019) Inflammasome Therapeutics - About Inflammasome Therapeutics.

59. Fowler BJ, Gelfand BD, Kim Y, Kerur N, Tarallo V, et al. (2014) Nucleoside reverse transcriptase inhibitors possess intrinsic anti-inflammatory activity. Science 346(6212): 1000-1003.

60. Zyversa.com. (2019) IC 100: ASC Inhibitor: ZyVersa Therapeutics Inc.

61. Wan H (2016) An Overall Comparison of Small Molecules and Large Biologics in ADME Testing. ADMET \& DMPK 4(1): 1.

62. Slastnikova T, Ulasov A, Rosenkranz A, Sobolev A (2018) Targeted Intracellular Delivery of Antibodies: The State of the Art. Frontiers in Pharmacology 9: 1208.

63. Nicholls SJ, Puri R, Anderson T, Ballantyne CM, Cho L, et al. (2016) Effect of evolocumab on progression of coronary disease in statin-treated patients: the GLAGOV randomized clinical trial. JAMA 316: 2373-2384.

64. Grebe A, Hoss F, Latz E (2018) NLRP3 Inflammasome and the IL-1 Pathway in Atherosclerosis. Circulation Research 122(12): 1722-1740.

65. Jin Y, Fu J (2019) Novel Insights Into the NLRP3 Inflammasome in Atherosclerosis. Journal of the American Heart Association 8(12): e012219.

66. Broz P, Dixit VM (2016) Inflammasomes: mechanism of assembly, regulation and signalling. Nat Rev Immunol 16: 407-420.
67. Mangan MSJ, Olhava EJ, Roush WR, Seidel HM, Glick GD, et al. (2018) Targeting the NLRP3 inflammasome in inflammatory diseases. Nature Reviews Drug Discovery 17(8): 588-606.

68. Emberson J, Bennett D (2006) Effect of alcohol on risk of coronary heart disease and stroke: causality, bias, or a bit of both. Vascular Health and Risk Management 2(3): 239-249.

69. Nurmi K, Virkanen J, Rajamäki K, Niemi K, Kovanen PT, et al. (2013) Ethanol Inhibits Activation of NLRP3 and AIM2 Inflammasomes in Human Macrophages-A Novel Anti-Inflammatory Action of Alcohol. PLoS ONE 8(11): e78537.

70. Clinicaltrials.gov. (2019) Interleukin-1 (IL-1) Blockade in Acute Myocardial Infarction (VCU-ART3) - Full Text View - ClinicalTrials.gov.

71. Clinicaltrials.gov. (2019) Cardiovascular Risk Reduction Study (Reduction in Recurrent Major CV Disease Events) - Full Text View ClinicalTrials.gov.

72. Clinicaltrials.gov. (2019) Rilonacept to Improve Artery Function in Patients With Atherosclerosis - Full Text View - ClinicalTrials.gov.

73. Issafras H, Corbin JA, Goldfine ID, Roell MK (2013) Detailed Mechanistic Analysis of Gevokizumab, an Allosteric Anti-IL-1 $\beta$ Antibody with Differential Receptor-Modulating Properties. Journal of Pharmacology and Experimental Therapeutics 348(1): 202-215.

74. Clinicaltrials.gov. (2019) Type 2 Diabetic Patients Maintained on Statin Therapy - Full Text View - ClinicalTrials.gov.

75. Clinicaltrials.gov. (2019) Colchicine in Vascular Inflammation Assessed With PET Imaging - Full Text View - ClinicalTrials.gov. 